\title{
A Year in Retrospect
}

\author{
Bhavin J ankharia \\ Editor-in-Chief, Indian J ournal of Radiology and Imaging, Bhaveswar Vihar, 383 Sardar V. P. Road, Prathana Samaj, \\ Mumbai - 400 004, India
}

Correspondence: Bhavin Jankharia, Editor-in-Chief, Indian Journal of Radiology and Imaging, Bhaveswar Vihar, 383 Sardar V. P. Road, Prathana Samaj, Mumbai - 400 004, India. E-mail: editor@ijri.org

This November issue has been very exciting to publish. This particular issue marks the end of our first year. We are proud that this fourth issue of 2007 has been available online from 31 October, and will have reached most radiologists in print form, in the month of November itself; all our endeavors are slowly bearing fruit.

The team is now working cohesively. Dr. Sanjay Jain, our Joint Editor and Secretary, has shouldered the administrative responsibilities admirably and along with Ms. Shamina Petiwala's help, has streamlined all our processes. Dr. Sanjeev Mani, one of our two Associate Editors, takes care of the website and related issues. The website, www.ijri.org is popular and has a daily viewership of approx. 4000 . Our Ahead of Print (AOP) feature has finally started as well and all accepted articles are now being put up in this section, irrespective of when they will be published. Dr. IK Indrajit, our second Associate Editor handles two sections in the journal, "Computers in Radiology" and "Web Reviews", both of which are becoming more and more popular.

The online submission system at www.journalonweb.com/ ijri, developed by Dr. D K Sahu of Medknow Publications, from scratch, has allowed us to move the entire article processing mechanism online. All authors register on the website and then upload their articles. Each article is first evaluated for its compatibility with the "Instructions for Authors". If all criteria have been met, then a quick assessment is made of whether it is worth processing further. $80 \%$ of all case reports are immediately rejected. To help in this process, we now have Internal Editors; Dr. Rakesh Gupta from SGPGI for neuroradiology and Drs. Nitin Chaubal from Mumbai and B. Ramamurthy from Bangalore for ultrasound help me in this process.

Once a decision is made to further process the article, it is then sent to Ms. Vasumathi Sriganesh and her team for a plagiarism check. If there is significant "lifting" and plagiarism, the article is sent back to the author for an explanation. If the explanation is credible, the author is allowed to rewrite the article. If the article comes back "clean" from the plagiarism check, it is then sent for External Review. National and international reviewers of repute are chosen for this purpose and are given 2 weeks for their reviews and comments. Once these come in, the article is re-evaluated in detail. These comments are then sent back to the author(s), so that he/they can revise the article. If the revised article is in keeping with the suggestions made by the reviewer(s), then the article is sent for copy-editing, then for proofs and then for publishing.

As you can see, the process is extremely involved and everything needs to work cohesively. A delay at any point can push back the time taken for processing significantly. As of 31 Oct, 2007, we have received 410 articles and our average time to publication is 4 months.

Our reviewers are our backbone, and we urge them to help us deliver the goods faster and in a more proficient manner. A good reviewer has to do the following: see if the article makes any contribution to literature, assess the scientific accuracy of the claims made in the article and look for areas where the authors can improve their delivery of content and ideas. Reviewers are not expected to do copy-editing; many reviewers believe that performing a grammatical and spelling check of the article constitutes a good review. This is not true. Copy-editing (grammar, spellings, syntax) is done by copy-editors; what we need from reviewers is their judgment on whether an article is worth publishing or not and if so, what are the changes that the authors need to make, to improve the quality of the article. If any reviewer does not have the time to review any one particular article, we would appreciate an early response, even if negative, so that we can then assign the article to someone else. Till date, we have had 332 reviewers, 149 of who are from India, and the rest from over 60 other countries.

This issue continues the musculoskeletal ultrasound minisymposium with three more articles. The February and May 2008 issues will focus on Interventional Radiology and the August and November 2008 issues on Obstetric Ultrasound.

As we move into the next year, many challenges face us. There are newer modalities, new paradigms to look at and learn from and regulatory challenges including the PNDT act, a possible service tax next year and the like.

We hope that this journal is able to serve as a forum for all such issues, apart from being the premier academic publication in this country. 